\title{
Branes With Extended Symmetry
}

\author{
Moshe Moshe* ${ }^{\dagger}$ \\ Physics Department, Technion, Haifa 32000 Israel \\ E-mail: imoshe@physics.technion.ac.īi \\ Norisuke Sakai \\ Physics Department, Tokyo Institute of Technology, Tokyo, 152-8581, Japan \\ E-mail: nssakaioth.phys.titech.ac.jpi
}

ABSTRACT: Brane actions possess first and second class constraints that result in local $\kappa$ symmetry. In a larger phase space the $\kappa$ symmetry of the D-particle (D0 brane) and the D1 brane is extended here into a larger symmetry by turning second class constraints into first class. Different gauge fixings result in different presentations of these systems while a "unitary" gauge fixing of the new symmetry retrieves the original action.

\section{Introduction}

Local $\kappa$ symmetry on the world-volume is an important ingredient in D-branes dynamics. The history of this symmetry goes back to the superparticle action [i] where it was

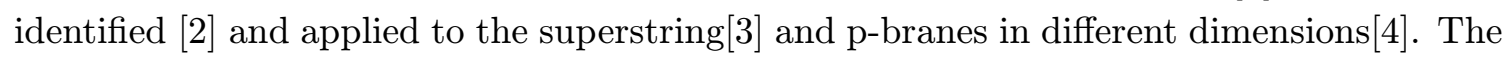
role of the $\kappa$ symmetry was further emphasized in the study of the D-branes embedded in

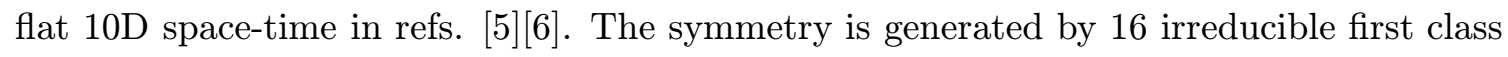
fermionic constraints. These constraints are accompanied by another set of 16 second class fermionic constraints which do not correspond to any local symmetry.

It has been found difficult to quantize covariantly the massless superparticle, as is the situation also with the Green-Schwarz formulation of the superstring [3in] since in both systems first and second class constraints cannot be separated in a covariant manner. This

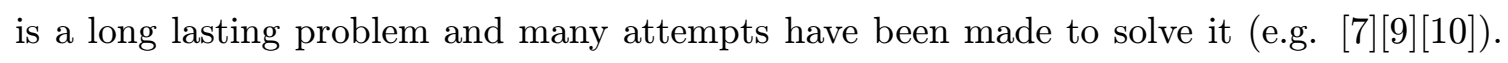
In the massive superparticle action the $\kappa$ symmetry is explicitly broken. Its first class constraints are replaced now by second class constraints and the system can be quantized covariantly by means of Dirac brackets since all its constraints are second class. Since the massive superparticle can be quantized covariantly, one may be tempted to consider the massless limit of the massive case as a substitute for the covariant quantization of the

${ }^{*}$ Speaker.

${ }^{\dagger}$ Summary of a talk at the European Physical Society Conference, Budapest July 2001 
massless superparticle. However, the Dirac brackets become singular in the $p^{2}=m^{2} \rightarrow 0$ limit. The restoration of the broken $\kappa$ symmetry of the massive system in an extended phase space [8] by adding extra fermionic degrees of freedom was considered in ref. [9]. The restoration of symmetry with no second class constraints, gives the full advantages of working within a system with local symmetry in particular a covariant wave function can be formulated also in the massless limit [ig]. For this purpose, it is usually useful to turn the second class constraints into first class. This formulation offers a flexibility to allow various gauge fixings which are physically equivalent. At the same time, the newly introduced first class constraints generate a gauge symmetry which may give more insight into the geometrical structure of the system which is interesting in its own right.

Several other different approaches to this issue share in common the idea of adding extra dynamical degrees of freedom while extending the symmetry of the system in different

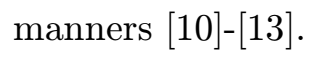

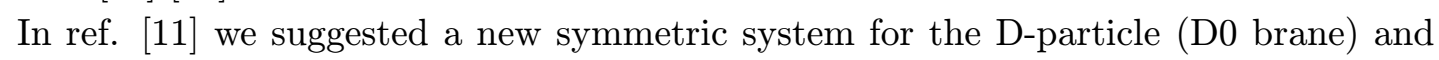
D1 brane in which the second class constraints are turned into first class in an extended phase space which includes extra fermionic degrees of freedom. A system was defined in [i] 1 in that contains $\theta_{\alpha}, \pi_{\alpha}$, the original fermionic degrees of freedom of the D0 brane to which extra fermionic degrees of freedom $\zeta_{\alpha}, \rho_{\alpha}$ are added $\left(\zeta_{\alpha}, \rho_{\alpha}\right.$ are Majorana-Weyl spinors while $\theta_{\alpha}, \pi_{\alpha}$ are only Majorana). The new system has, in addition to the original $\kappa=\kappa_{-}$symmetry a new local $\kappa_{+}$symmetry. The system can be gauge fixed in many different ways while one of these gauge fixings ("unitary" gauge) retrieves the original D0 brane. We also presented in [i] 1 in along the same lines, the D1 brane with an extended $\kappa_{-}$and $\kappa_{+}$local symmetry. We considered the case of a vanishing electric field in the Born-Infeld-Nambu-Goto action, (namely, $(0,1)$ string ).

\section{Superparticle and D-particle}

Local $\kappa$ symmetry is explicitly broken in the $\mathrm{N}=1$ massive superparticle action in $\mathrm{d}=10$ dimensions $[\overline{9} \overline{9}]$ :

$$
S=\int_{\tau_{i}}^{\tau_{f}} \mathcal{L}(\tau) d \tau=\int_{\tau_{i}}^{\tau_{f}} d \tau\left\{-\frac{1}{2 e}\left(\dot{x}^{\mu}-i \overline{\theta_{+}} \Gamma^{\mu} \dot{\theta_{+}}\right)^{2}+\frac{1}{2} e m^{2}\right\}
$$

Under the local $\kappa_{-}$transformation:

$$
\delta x_{\mu}=i \bar{\theta}_{+} \Gamma_{\mu} \delta \theta_{+} \quad, \quad \delta \theta_{+}=\left(\dot{x}^{\nu}-i \bar{\theta}_{+} \Gamma^{\nu} \dot{\theta}_{+}\right) \Gamma_{\nu} \kappa_{-} \quad, \quad \delta e=4 i e \dot{\bar{\theta}}_{+} \kappa_{-}
$$

one finds $\delta \mathcal{L}=2 \mathrm{iem}^{2} \dot{\bar{\theta}}_{+} \kappa_{-} \neq 0$ (for detailed notations see ref.[1] $\left.\overline{1}_{-1}\right)$ ). All 16 constraints are second class and its phase space has $(32-16=) 16$ independent fermionic degrees of freedom. A possible modification by which the local $\kappa_{-}$symmetry can be restored is extending its phase space to $\mathrm{N}=2$ while adding an appropriate Wess-Zumino term.

$$
\mathcal{L}=-\frac{1}{2 e}\left(\dot{x}^{\mu}-i \bar{\theta} \Gamma^{\mu} \dot{\theta}\right)^{2}+\frac{1}{2} e m^{2}+\mathcal{L}_{2}
$$

Here $\theta=\theta_{+}+\theta_{-}\left(\theta\right.$ is a Majorana spinor and $\theta_{+}$and $\theta_{-}$are Majorana-Weyl spinors of opposite chirality) and $\mathcal{L}$ in Eq.(高㥵) has a restored $\kappa_{-}$symmetry. The system has now 
not only 16 first class constraints but also 16 second class constraints and the number of independent degrees of freedom in phase space is the same as the $\mathrm{N}=1$ massive superparticle $(64-2 \times 16-16=16)$

A very appealing point of view on $\mathcal{L}(\tau)$ of Eq.(1) $\left(\begin{array}{l}1 \\ 1\end{array}\right)$ is obtained when one starts with the

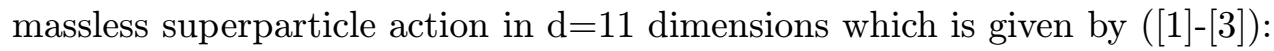

$$
S=\int_{\tau_{i}}^{\tau_{f}} \mathcal{L}(\tau) d \tau=-\frac{1}{2} \int_{\tau_{i}}^{\tau_{f}} d \tau e^{-1}\left(\dot{x}^{\hat{m}}-i \bar{\theta} \Gamma^{\hat{m}} \dot{\theta}\right)^{2}
$$

where $x^{\hat{m}}(\hat{m}=0,1 \ldots 10)$ are the space-time coordinates and $\theta_{\alpha}=\theta_{+\alpha}+\theta_{-\alpha}(\alpha=1,2 \ldots 32)$ are the corresponding fermionic coordinates which can be regarded as two Majorana Weyl spinors of opposite chiralities, if viewed as spinors in ten dimensions.

When one of the space directions is compactified to a radius of $R=m^{-1}=Z^{-1}$, the $\mathrm{d}=11$ massless superparticle action results [i6] in the D0 brane action.

$$
\begin{aligned}
S=\int_{\tau_{i}}^{\tau_{f}} \mathcal{L}(\tau) d \tau=\int_{\tau_{i}}^{\tau_{f}} d \tau\left\{\frac{1}{2 e}\left(\dot{x}^{\mu}-i \bar{\theta} \Gamma^{\mu} \dot{\theta}\right)^{2}+\frac{1}{2} e Z^{2}-i Z \bar{\theta} \Gamma^{11} \dot{\theta}\right\} \\
+Z\left[x_{10}\left(\tau_{f}\right)-x_{10}\left(\tau_{i}\right)\right]
\end{aligned}
$$

Where $p_{10}$ was set to $p_{10}=m=Z, \Gamma^{\hat{10}}$ is defined as $\Gamma^{11}$ and $\mu=0,1 \ldots 9$. The D0

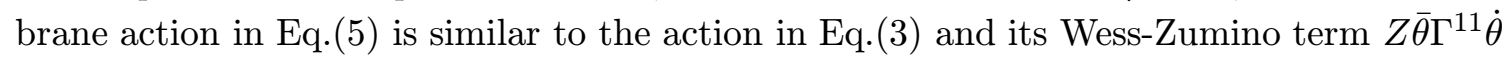
establishes the local $\kappa_{-}$symmetry, which is the original symmetry of the $\mathrm{d}=11$ massless superparticle action. Thus, instead of 32 second class constraints as in the $\mathrm{N}=2, \mathrm{~d}=10$ massive superparticle action, the D0 has 16 first class constraints and 16 second class constraints which is the same number of constraints as the massless $N=2, d=10$ superparticle and here too the 16 first class constraints result in $\kappa_{-}$symmetry. An important difference between the D0 action and the massless superparticle is the fact that in the D0 case the first and second class constraints can be separated in a covariant manner [i, $\mathbf{i}]$, this cannot be done for the massless $\mathrm{N}=2 \mathrm{~d}=10$ superparticle.

In ref [i 11 in we treated the D0 system in a more symmetrical manner by turning also its remaining 16 second class constraints into first class. The resulting system has in addition to the original $\kappa_{-}$symmetry also a $\kappa_{+}$symmetry generated by the new first class constraints.

The first and second class constraints that result from the action in Eq. Eq.(鼠) can be

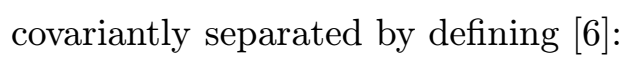

$$
\begin{aligned}
& \bar{T}_{1}=\bar{T}\left(\not p+Z \Gamma^{11}\right)\left(\frac{1-\Gamma^{11}}{2}\right)=\bar{\pi}_{-} \not p-Z \bar{\pi}_{+}+i \bar{\theta}_{+}\left(p^{2}+Z^{2}\right) \\
& \text { and } \\
& \bar{T}_{2}=\bar{T}\left(\frac{1+\Gamma^{11}}{2}\right)=\bar{\pi}_{-}+i \bar{\theta}_{+} \not p+i Z \bar{\theta}_{-}
\end{aligned}
$$

as seen from their Poisson bracket relations ( we also have the constraint $p^{2}+Z^{2}=0$ ).

The extended phase space is now defined by adding $\rho_{-}$and $\zeta_{+}$, a canonical pair of Majorana-Weyl spinors representing extra 32 fermionic degrees of freedom whose Poisson bracket is: $\left[\bar{\rho}_{-\alpha}, \zeta_{+\beta}\right]=\frac{1}{2}\left(1+\Gamma^{11}\right)_{\alpha \beta}$. The dynamics in the extended phase space is defined by the two opposite chirality sets of constraints $\bar{T}_{+}, \bar{T}_{-}^{\prime}$ and their Poisson bracket:

$$
\bar{T}_{1} \equiv \bar{T}_{+}=\bar{\pi}_{-} \not p-Z \bar{\pi}_{+}+i \bar{\theta}_{+}\left(p^{2}+Z^{2}\right) \quad, \quad \bar{T}_{-}^{\prime}=\bar{\pi}_{-}+i \bar{\theta}_{+} \not p+i Z \bar{\theta}_{-}-i \bar{\rho}_{-}+\bar{\zeta}_{+} \not \supset .
$$


The Poisson brackets of the two chiral multiplets of these first class constraints in the extended phase space are:

$$
\left[\bar{T}_{+\alpha}, \bar{T}_{+\beta}\right]=-2 i\left(p^{2}+Z^{2}\right)\left(\Gamma^{0}\left(\frac{1+\Gamma^{11}}{2}\right) \not p\right)_{\alpha \beta},\left[\bar{T}_{+\alpha}, \bar{T}_{-\beta}^{\prime}\right]=-2 i\left(p^{2}+Z^{2}\right)\left(\Gamma^{0}\left(\frac{1+\Gamma^{11}}{2}\right)\right)_{\alpha \beta}
$$

and $\left[\bar{T}_{-\alpha}^{\prime}, \bar{T}_{-\beta}^{\prime}\right]=0$. The total extended phase space hamiltonian is $H_{T}=H_{0}+\frac{1}{2} \lambda_{p}\left(p^{2}+\right.$ $\left.Z^{2}\right)+\bar{T}_{+} \lambda_{-}+\bar{T}_{-}^{\prime} \lambda_{+}$, where $H_{0}=-\frac{1}{2} e\left(p^{2}+Z^{2}\right)$. The generator of $\kappa_{-}$and $\kappa_{+}$gauge symmetries and reparametrization is: $G=\epsilon_{e} \pi_{e}+\frac{\epsilon_{p}}{2}\left(p^{2}+Z^{2}\right)+\left\{\bar{\pi}_{-} \not p-Z \bar{\pi}_{+}+i \bar{\theta}_{+}\left(p^{2}+\right.\right.$ $\left.\left.Z^{2}\right)\right\} \kappa_{-}+\left\{\bar{\pi}_{-}+i \bar{\theta}_{+} \not p+i Z \bar{\theta}_{-}-i \bar{\rho}_{-}+\bar{\zeta}_{+} \not p\right\} \kappa_{+}$

We started with 16 fermionic degrees of freedom in phase space $(64-16 \times 2-16)$, extra 32 degrees of freedom $\left(\rho_{-}, \zeta_{+}\right)$were added and the $\kappa_{+}$symmetry was introduced. We have now 16 independent degrees of freedom as in the original system $(64+32-16 \times 2-16 \times 2=$ $16+16_{\text {decoup. }}$ ) while the other $16_{\text {decoup }}$. are the "Batalin-Fradkin decoupled" [1] 1 in degrees of freedom.

In the extended symmetry system, in addition to the possible gauge fixing (e.g. [']b]) that eliminates the $\theta_{-}$degrees of freedom by fixing the $\kappa_{-}$gauge, other gauge fixings are acceptable as well. Clearly, a properly chosen gauge fixing ("unitary" gauge fixing) of the new $\kappa_{+}$symmetry will eliminate the linear combination of the new fermionic degrees of freedom $-i \bar{\rho}_{-}+\bar{\zeta}_{+} \not p$. For example a possible unitary gauge fixing is $\theta_{-}=0$ and $-i \bar{\rho}_{-}+$ $\bar{\zeta}_{+} \not \supset=0$. This results in the same gauge fixed system that was used in [i]"]. A different, interesting, gauge fixing that eliminates the old degrees of freedom and leaves only the new 16 degrees of freedom is simply, $\theta_{-}=0$ and $\theta_{+}=0$. The gauge fixed D0 system is given in this gauge in terms of the new coordinates $-i \bar{\rho}_{-}+\bar{\zeta}_{+} \not p$ only. As in the case of the unitary gauge the Poisson bracket matrix $\left[\bar{T}_{ \pm \alpha}, \chi_{ \pm \beta}\right]$ between the constraints $\bar{T}_{+\alpha}, \bar{T}_{-\alpha}^{\prime}$ and the gauge fixing conditions $\chi_{-}=\theta_{-}, \chi_{+}=\theta_{+}$is not singular since $p^{2}+Z^{2}=0$. Of course, other combinations of $\kappa_{-}$and $\kappa_{+}$gauge fixings are also possible.

\section{D1 brane with $\kappa_{-}$and $\kappa_{+}$extended symmetry}

Following along similar lines we presented in [i]1, the extension of this derivation to the case of a D1 brane. It results in a system with $\kappa_{-}$and $\kappa_{+}$symmetry. The action of the D1 brane consists of the Born-Infeld-Nambu-Goto term and the Chern-Simons two form

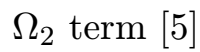

$$
S=\int \mathcal{L}(\sigma) d^{2} \sigma=-T\left\{\int d^{2} \sigma \sqrt{-\operatorname{det}\left(G_{\mu \nu}+\mathcal{F}_{\mu \nu}\right)}+\int \Omega_{2}\right\}
$$

where $G_{\mu \nu}$ is the supersymmetric induced world-volume metric and $\mathcal{F}_{\mu \nu}$ is the supersymmetric Born-Infeld field strength (we will suppress the indices $A=1,2$ of $\theta_{\alpha}^{A}$ when it is easily recognized). From Eq. $\left(\bar{G}^{\prime} \bar{l}\right)$ one finds the fermionic constraints $\bar{\Phi}_{\alpha}^{A}$

$$
\bar{\Phi}_{\alpha}=\bar{\pi}_{\alpha}+(\bar{\theta} \not \partial)_{\alpha}-\left(\bar{\theta} \Gamma^{m} T_{E}\right)_{\alpha}\left(\partial_{1} x_{m}\right)+\left(\bar{\theta} \Gamma^{m} \partial_{1} \theta\right)\left(\bar{\theta} \Gamma_{m} T_{E}\right)_{\alpha}=0
$$

where $T_{E}=E^{1} \tau_{3}+T \tau_{1}$ ( $E^{1}$ is the electric field and $\tau_{k}$ are Dirac matrices). The constraints in Eq.(i $\left.{ }^{i} \overline{0}_{-}^{\prime}\right)$ can be separated covariantly into first class and second class constraints:

$$
\bar{T}_{1 \alpha}=\left(\bar{\Phi}\left(\tilde{p}-\not h T_{E}\right)\left(\frac{1+\tau_{3}}{2}\right)\right)_{\alpha} \quad, \quad \bar{T}_{2 \alpha}=\left(\bar{\Phi}\left(\frac{1-\tau_{3}}{2}\right)\right)_{\alpha}
$$


The Poisson bracket $\left[\bar{T}_{1 \alpha}, \bar{T}_{1 \beta}\right]$ vanishes on the constraints hyperplane. These 16 first class constraints $\bar{T}_{1 \alpha}$ generate the local $\kappa$ symmetry of the D1 brane. On the other hand $\bar{T}_{2 \alpha}$ are 16 second class constraints $\left[\bar{T}_{2 \alpha}, \bar{T}_{2 \beta}\right]=2\left(\Gamma^{0} \not P \tau_{-}\right)_{\alpha \beta} \delta\left(\sigma-\sigma^{\prime}\right)$ where $P_{m}=\tilde{p}_{m}+E^{1} \Pi_{1 m}=$ $p_{m}+\bar{\theta} \Gamma_{m} T_{E} \partial_{1} \theta+E^{1}\left(\partial_{1} x_{m}-\bar{\theta} \Gamma_{m} \partial_{1} \theta\right)$

The condition $G_{11} \neq 0$ is essential for separating the first and second class constraints and the covariant quantization of the D1 system. In Ref.[i[i]] it has been emphasized that in the static gauge (where $x^{\mu}=\sigma^{\mu}$ for $\mu=0,1$ ) indeed $G_{11} \neq 0$ and the implications of this fact on the ground state spectrum was discussed there. Since the static gauge is a natural gauge for D1, we follow this point of view.

We defined in [1] $\left.\mathbf{1}_{1}^{1}\right]$ the D1 brane system in an extended phase space that includes in addition to the 64 fermionic degrees of freedom $\theta_{\alpha}^{A}$ and $\pi_{\alpha}^{A}$ extra fermionic 32 degrees of freedom that satisfy $\left[\bar{\rho}_{\alpha}^{A}(\sigma), \zeta_{\beta}^{B}\left(\sigma^{\prime}\right)\right]=\delta\left(\sigma-\sigma^{\prime}\right) \tau_{-}^{A B} \delta_{\alpha \beta}$ The constraints of this new D1 system $\bar{T}_{\alpha}^{\prime}{ }^{A}(x, p, \theta, \pi, \zeta, \rho)$ were obtained in a similar way the constraints in the extended phase space for the Dparticle. Namely, $\bar{T}_{1 \alpha}^{\prime A}(x, p, \theta, \pi, \zeta, \rho)=\bar{T}_{1 \alpha}^{A}(x, p, \theta, \pi)$ is left unchanged and does not depend on $(\zeta, \rho)$ whereas the other constraint $T_{2 \alpha}^{A}$ is modified as

$$
\bar{T}_{2 \alpha}^{\prime A}(x, p, \theta, \pi, \zeta, \rho)=\bar{T}_{2 \alpha}^{A}(x, p, \theta, \pi)-\bar{\rho}_{\alpha}^{A}+\left(\bar{\zeta}^{B} \not P\right)_{\alpha} \tau_{-}^{B A}
$$

which depends on $(\zeta, \rho)$ and satisfies the Poisson bracket relation:

$$
\begin{array}{r}
{\left[\bar{T}_{2 \alpha}^{\prime}, \bar{T}_{2 \beta}^{\prime}\right]=-2 E^{1} \delta\left(\sigma-\sigma^{\prime}\right)\left(2\left(\Gamma^{0} \Gamma^{m}\right)_{\alpha \beta}\left(\bar{\zeta} \Gamma_{m} \tau_{-} \partial_{1} \theta\right)-\left(\bar{\zeta} \Gamma^{m}\right)_{\alpha}\left(\partial_{1} \bar{\zeta} \Gamma_{m} \tau_{-}\right)_{\beta}\right)} \\
-2 E^{1} \frac{\partial \delta\left(\sigma-\sigma^{\prime}\right)}{\partial \sigma^{\prime}}\left(\bar{\zeta} \Gamma^{m}\right)_{\alpha}\left(\bar{\zeta} \Gamma_{m} \tau_{-}\right)_{\beta}
\end{array}
$$

In the case of $E^{1}=0$ the new system has only first class constraints and local symmetries $\kappa_{1}$ and $\kappa_{2}$ generated by $\bar{T}_{1 \alpha}$ and by $\bar{T}_{2 \alpha}^{\prime}$ respectively. The symmetric system phase space is given by the coordinates $\theta_{\alpha}^{A}(\sigma), \pi_{\alpha}^{A}(\sigma), \rho_{\alpha}^{A}(\sigma)$ and $\bar{\zeta}_{\beta}^{B}(\sigma)$ where the number of independent fermionic degrees of freedom has not been changed. Namely, we started with $2 \times 32-2 \times$ $16-16=16$ independent fermionic degrees of freedom in phase space and in the extended phase space we have $3 \times 32-2 \times 32=16+16_{\text {decoup. }}$ degrees of freedom where the $16_{\text {decoup }}$. degrees of freedom are "Batalin Fradkin decoupled" [9]9] leaving 16 independent fermionic degrees of freedom.

We noted that setting $E^{1}=0$ means also that $\mathcal{F}_{01}=0$ which results in the Lagrangian of Eq. $\left(\bar{p}_{1}^{1}\right)$ ) to be very similar to the Green-Schwarz string. Namely, using the equation of motion one notices that the D1 action in Eq.(高) with $E_{1}=0$ is identical to the GreenSchwarz action when $\tau_{3}$ is replaced by $\tau_{1}$. Since we are using the static gauge as a natural gauge for D1 [5] ne the massless modes are projected out. This relates the physics of the type IIB fundamental string and the D1 system in the static gauge [īi]. We also note that the electric field $E^{1}$ is quantized and represents the number of fundamental string bound

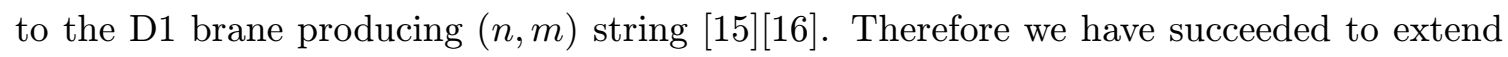
the system where all the second class constraints are turned into first class constraints at least for the case of the $(0,1)$ string, namely the genuine D1 brane without F1 provided the massless modes are projected out by using, for instance, the static gauge.

\section{Work supported in part by the Israeli Science Foundation and the Technion VPR fund.}




\section{References}

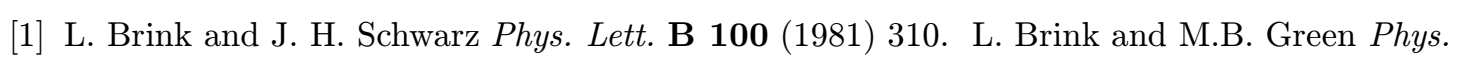

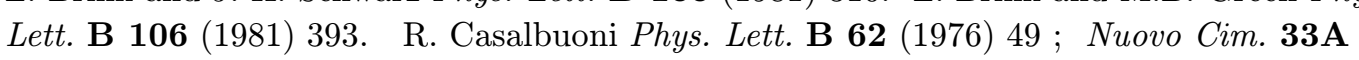
$(1976) 389$.

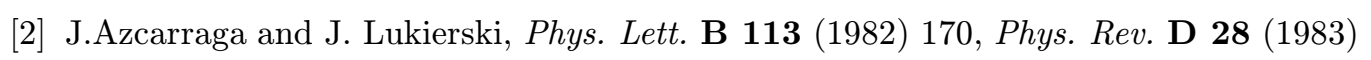

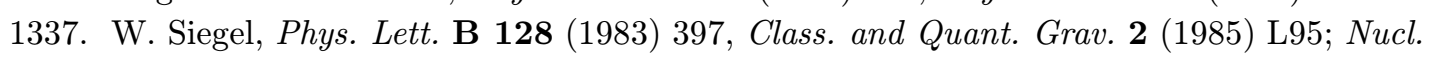
Phys. B 263 (1986) 93 :

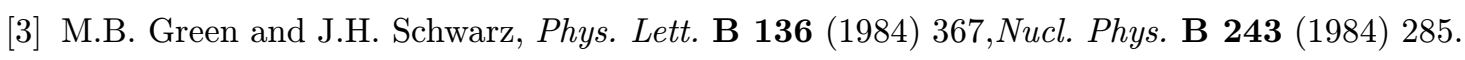

[4] J. Hughes, J. Liu, and J. Polchinski Phys. Lett. B $180-1986) 370$. A detailed review on superembeding and references to earlier works is found in: D. Sorokin "Phys. Rept. $\mathbf{3} 2 \mathbf{9} 9(2000)$ (1, hep-th/9906142:

[5] M. Aganagic, C. Popescu, J. H. Schwarz iNucl. Phys. B 495 (1997) 99, hepeth/9612080; PPhys. Lett. B 393 (1997) 311, hep-th/9610249, E. Bergshoeff and P.K. Townsend 'Nucl.' 1. - Phys. B 490 $(1997) 145$, hep-th/9611173

[6] R. Kallosh, 'Phys. Rev. D 56 (1997) 35151, hep-th/9705056! M. Hatsuda and K. Kamimura iNucl. Phys. B $520(1998) 335$ hep-th/9708001!

[7] A. Mikovic, M. Rocek, W. Siegel, P. van Nieuwenhuizen, J. Yamron and A.E. van de Ven, Phys. Lett. B235, 106 (1990) Phys. Lett. B 235 $(1990) 106 ;$ F. Essler, M. Hatsuda,

E. Laenen, W. Siegel, J.P. Yamron, T. Kimura and A. Mikovic, 'Nucl. Phys. B-364 (1991) $6 \overline{1}$. W. Siegel,

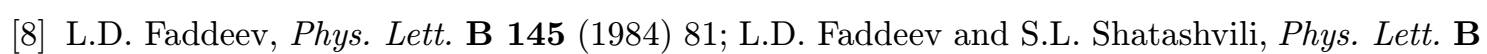

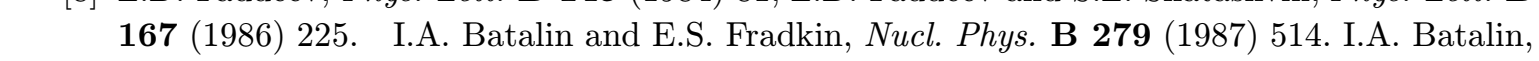
E.S. Fradkin and T.E. Fradkina iNucl. Phys. B 314 (1989) 158.

[9] M. Moshe Proc. of 11th Workshop on Current Problems in Particle Theory. Published in Johns Hopkins Workshop 1987:0207 (QCD161:J55:1987). J. Feinberg and M. Moshe, 'Ṕ

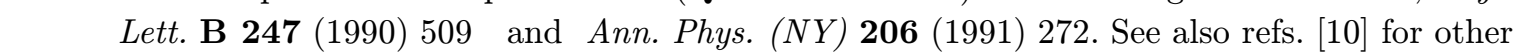
applications of 8

[10] Y. Eisenberg and S. Solomon 'Nucl. Phys. D. Sorokin Phys. Rev. D61 (2000) 45002, hep-th/9904109.

[11] M. Moshe and N. Sakai, Phys. Rev. D2 62000$) 86004$ hep-th/9912043i.

[12] I.A. Bandos and A.A. Zheltukhin Phys. Lett. B $288(1992) 77$.

S. Aoyama, P.Pasti and M. Tonin 'Phys. Lett. B 283 (1992) 213

N. Berkovits iNucl. Phys. B $43 \overline{1}(1999 \overline{4}) 258$.

[13] A. Duncan and M. Moshe Gitman iNucl. Phys. B $536(1999) 435$

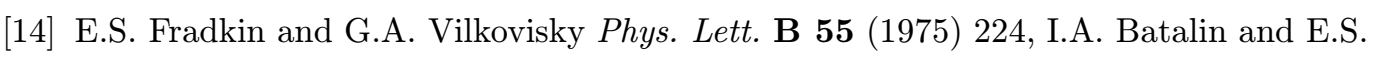
FradkiniPhys. Lett. B 122 19832 15t,Phys. Lett. B 128 1983 303

[15] E. Witten, 'Nucl. Phys. B 460_(1996) 493, 'hep-th/9510135'.

[16] J. Polchinski, String Theory, Vol.II,sect.13.6, Cambridge Univ. Press (1998) 